\title{
THE FORMATION OF DISKS BY INELASTIC COLLISIONS OF GRAVITATING PARTICLES. APPLICATIONS TO THE DYNAMICS OF THE SATURN'S RING AND TO THE FORMATION OF THE SOLAR SYSTEM
}

\author{
A. B RAHIC
}

Observatoire de Paris-Meudon, Université de Paris VII, France

\begin{abstract}
We integrate numerically the evolution of a three-dimensional system of particles with finite dimensions, which bounce inelastically upon each other. The particles are subjected to the attraction of a central mass; their mutual attraction is neglected. This model is used to study the evolution of Saturn's ring. The first results are presented: such a collision mechanism can flatten very quickly the Saturn's ring and the system tends towards a final equilibrium state.
\end{abstract}

Collisions between macroscopic objects are surely a common feature of the Universe: these objects may range from interstellar clouds to solid bodies in the solar system. We would like to know in what way such collisions have affected the evolution of the system in which they occur.

Poincare (1911) showed qualitatively that, in a nebula consisting of a number of bodies undergoing inelastic collisions, a central condensation will be formed, the system as a whole will flatten into a plane perpendicular to the initial angular momentum vector and the orbits of the bodies will become more circular.

Since the time of Descartes, Laplace and Poincaré, the idea of a primordial chaotic nebula has played a central role in cosmogonic speculation.

Many researchers invoke collisions between discrete bodies or 'flocules' to explain the formation of the solar system 'disk' and of the planets (MacCrea, 1960; Alfvén and Arrhenius, 1970a, b; Urey, 1972).

Collisions have certainly played an essential role in the formation and evolution of Saturn's ring (Jeffreys, 1947; Cook et al., 1972). Many critical questions still remain unanswered:

Is the ring a few thousand millions years old and was it, in that case, created from circulating material which was flattened through inelastic collisions and which could not condense into a satellite because it is within the Roche limit? We may in this case ask why the ring does not extend uniformly to the planet itself.

Or is the ring younger than this and the debris of a satellite disrupted by tidal forces? Now, if such a satellite were to pass near to Saturn on an hyperbolic orbit, it would certainly be broken up, but in this case the pieces would not remain around the planet. It is difficult to envisage a satellite spiralling inward since, in this particular case, tidal forces would tend rather to push the satellite out. Note that if a satellite did get broken up inside the Roche limit and the debris remained in orbit, then since the rocks have some degree of cohesion, their dimensions would be about one hundred times the observed thickness of the ring (Bobrov, 1970). 
Does the ring consist of one layer of bodies, or more? Since the last century, many researchers have suggested that the luminosity of the rings as a function of their inclination indicates the presence of several layers which throw shadows on each other; on the other hand, collisions would rapidly reduce the system to one layer only. It has been shown in the case of the lunar surface that one layer of material could be sufficient to generate such a 'cat's eye' effect.

What is the characteristic size of the material? What is its nature? Is it frost, boulders or anything else...?

There has been some analytic analysis made for systems of orbiting colliding particles, based on the Boltzmann equation, but in view of the approximations which have to be introduced, its usefulness in the present context is debatable.

With the help of Michel Hénon, I am at present developing a general programme for this kind of problem. It is a numerical simulation of a gravitating system of particles with inelastic collisions. Such a programme has many potential applications, for instance the dynamics of Saturn's ring, the formation of the solar system, the evolution of the nuclei of galaxies (Spitzer and Saslaw, 1966; Spitzer and Stone, 1967; Sanders, 1970), the formation of galaxies (Brosche, 1970).

Calculations of this kind have so far only been carried out by Ulam (1968), who was interested in the nuclei of galaxies, and by Trulsen (1972a, b, c), who studied the dynamics of jet streams and Saturn's ring. Corresponding numerical experiments (Alder and Wainwright, 1959, 1960; Rahman, 1964; Verlet, 1967, 1968) have simulated significant progress in molecular dynamics.

The technical details of the rather intricate calculations will be given in a paper in preparation. The principal difficulty is to know whether two particles will in fact collide.

A very similar approach has also been used by us in the case of the formation of galaxies. The results are presented elsewhere (Brahic, 1973).

The time scale $t_{c}$ of the evolution of the systems is of the order of the mean time between collisions for one particle. Therefore, it is inversely proportional to the number $N$ of particles and to their geometrical cross-section, which is itself proportional to the square of the radius $R$ of a particle.

In fact, if we change either $N$ or $R$, we simply make the evolution go slower and faster. Consequently, a proper choice for $N$ and $R$ permits us to follow realistically the evolution of our system using a minimum of computer time.

Results have been obtained for the simplest case in which particle orbits are Keplerian around a central mass point. Positions and velocities at any given time are obtained from Kepler's equation. In a collision, the normal component is multiplied by a coefficient $k$ which lies between 0 and 1 .

The kinetic rotational energy of the bodies has been neglected. Each collision is assumed to be instantaneous.

The initial conditions were set up by selecting at random the six elements of the Keplerian orbit in such a way that trajectories were all ellipses lying between two spheres centred on the central mass point and with inclinations lying between 0 and 
some maximum value. We have assumed that clouds on hyperbolic trajectories escape at once and, for technical reasons, that clouds near to the center are captured by the center of mass.

In our experiments, a particle undergoes on the average one collision per revolution: a higher frequency and therefore a smaller mean free path would change the
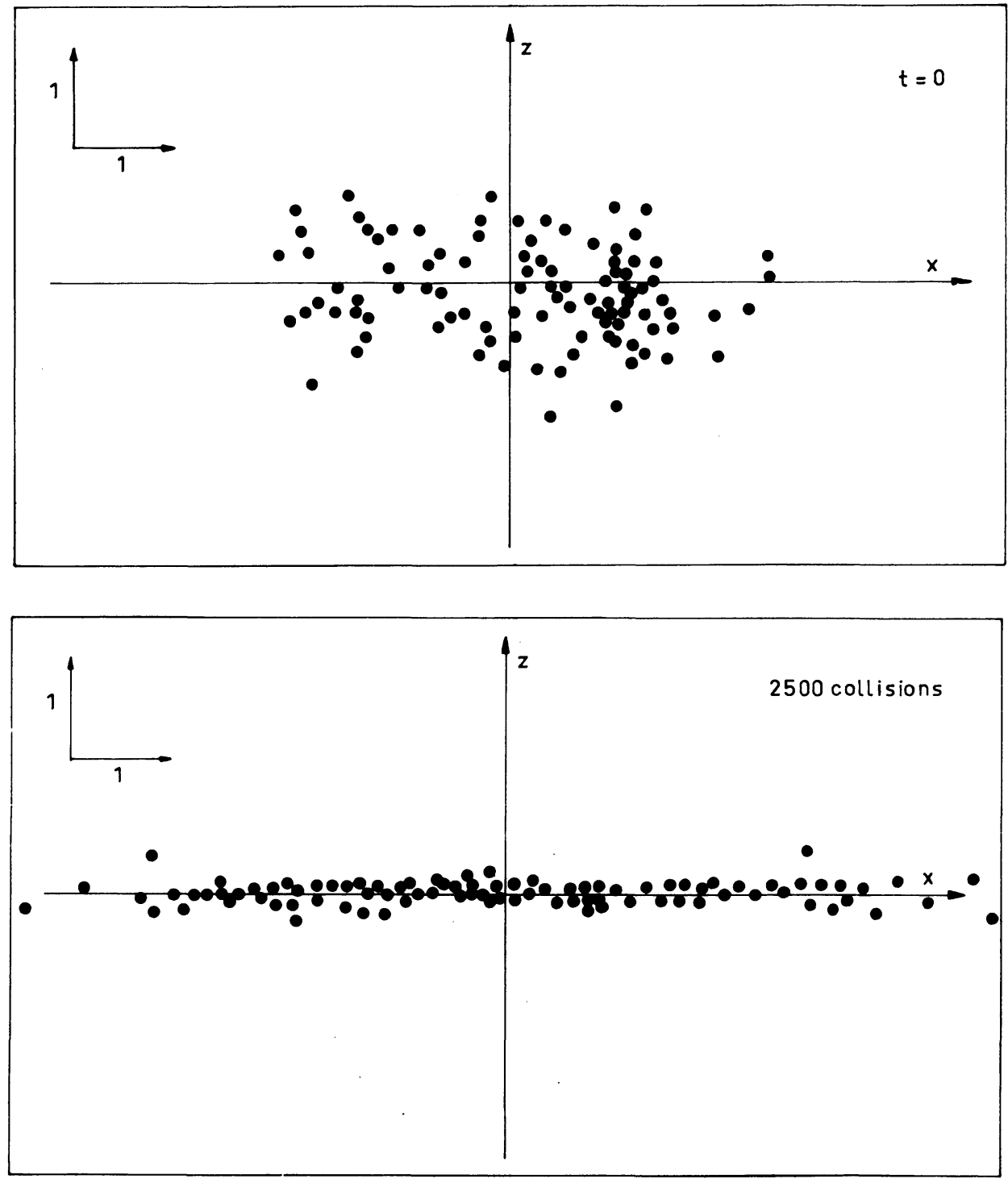

Fig. 1. Projections onto a plane parallel to the initial angular momentum vector at the initial time and at the end of the computation. The number $N$ of clouds is equal to 100. Each cloud has the same radius $R=0.07$ and the same mass. The coefficient of elasticity $k$ is equal to 0.1 . The maximum value of the initial inclinations of the orbits is equal to $0.5 \mathrm{rad}$. After 2500 collisions, no cloud has escaped and twelve out of 100 clouds have fallen on the central body of radius 0.1 . 

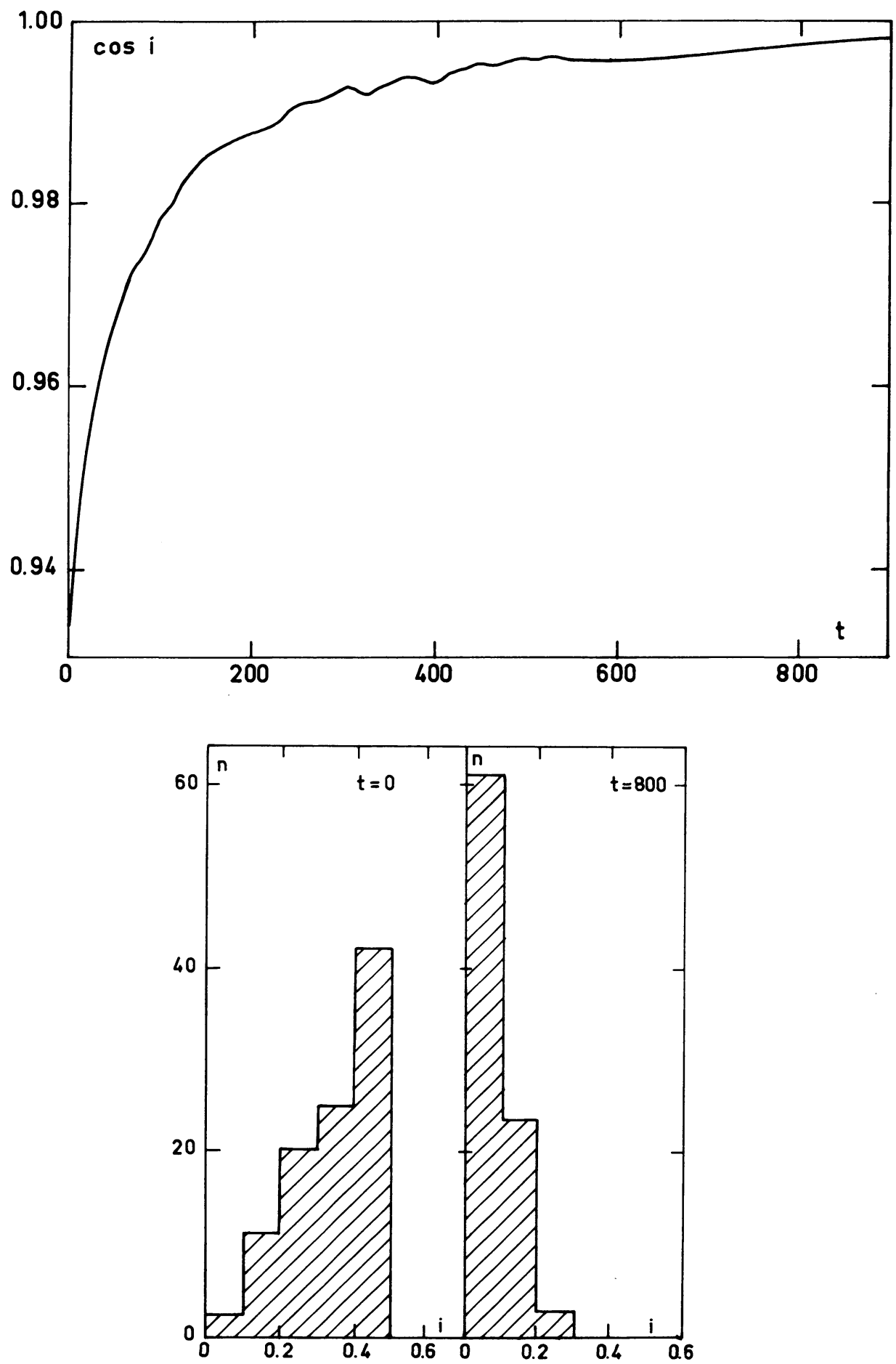

Fig. 2. Top: the variation as a function of time of the mean cosines of the inclination $i$. Bottom: the histogram of $i$ at the initial time and at the end of the computation. The unit of time corresponds approximately to the mean time necessary to one cloud to turn of one radian on its orbit. $k=0.1$. 

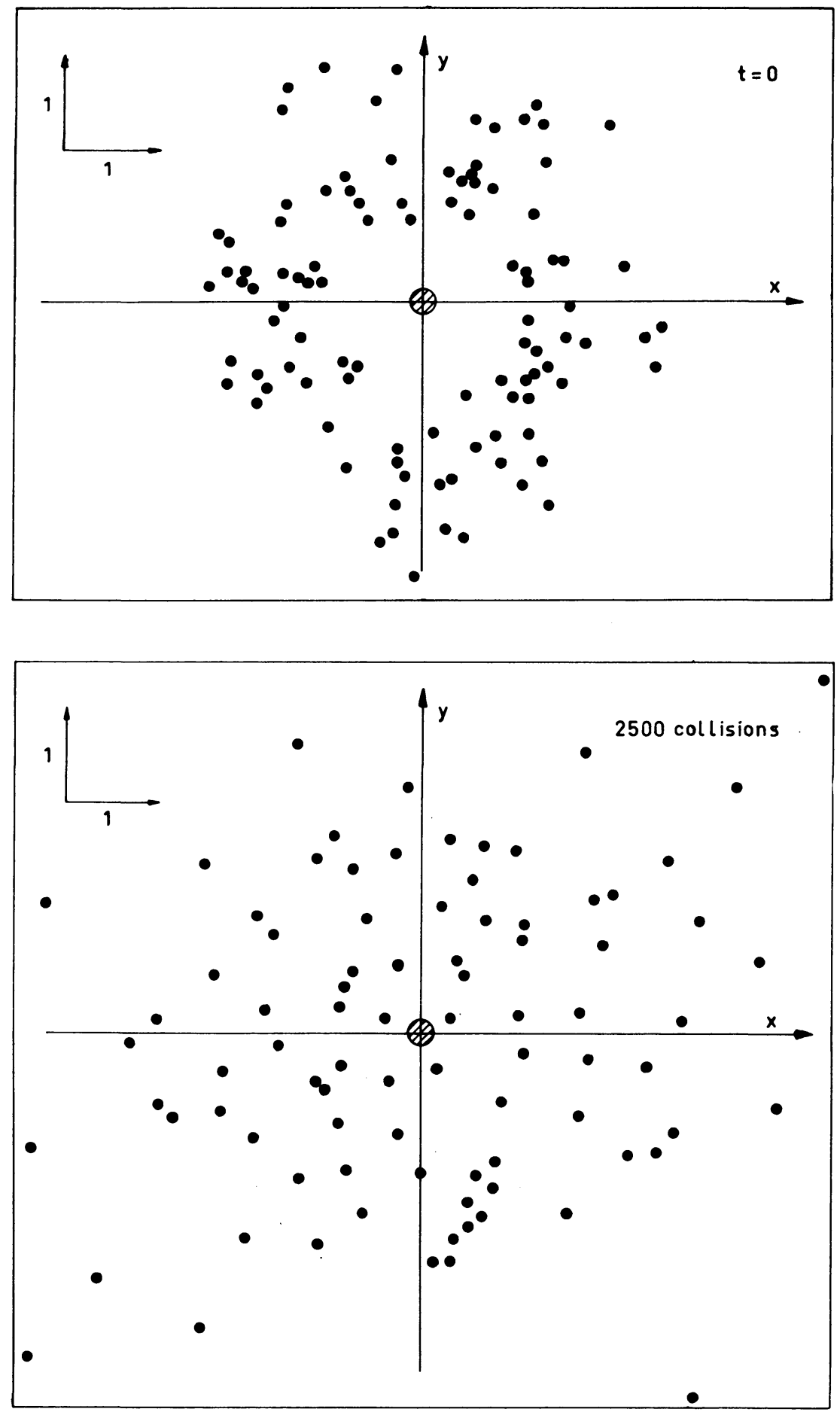

Fig. 3. Projections onto a plane perpendicular to the initial angular momentum vector. Initial trajectories are all ellipses lying between two spheres of radius 1 and 3 respectively centred on the central mass point. $k=0.1$. 

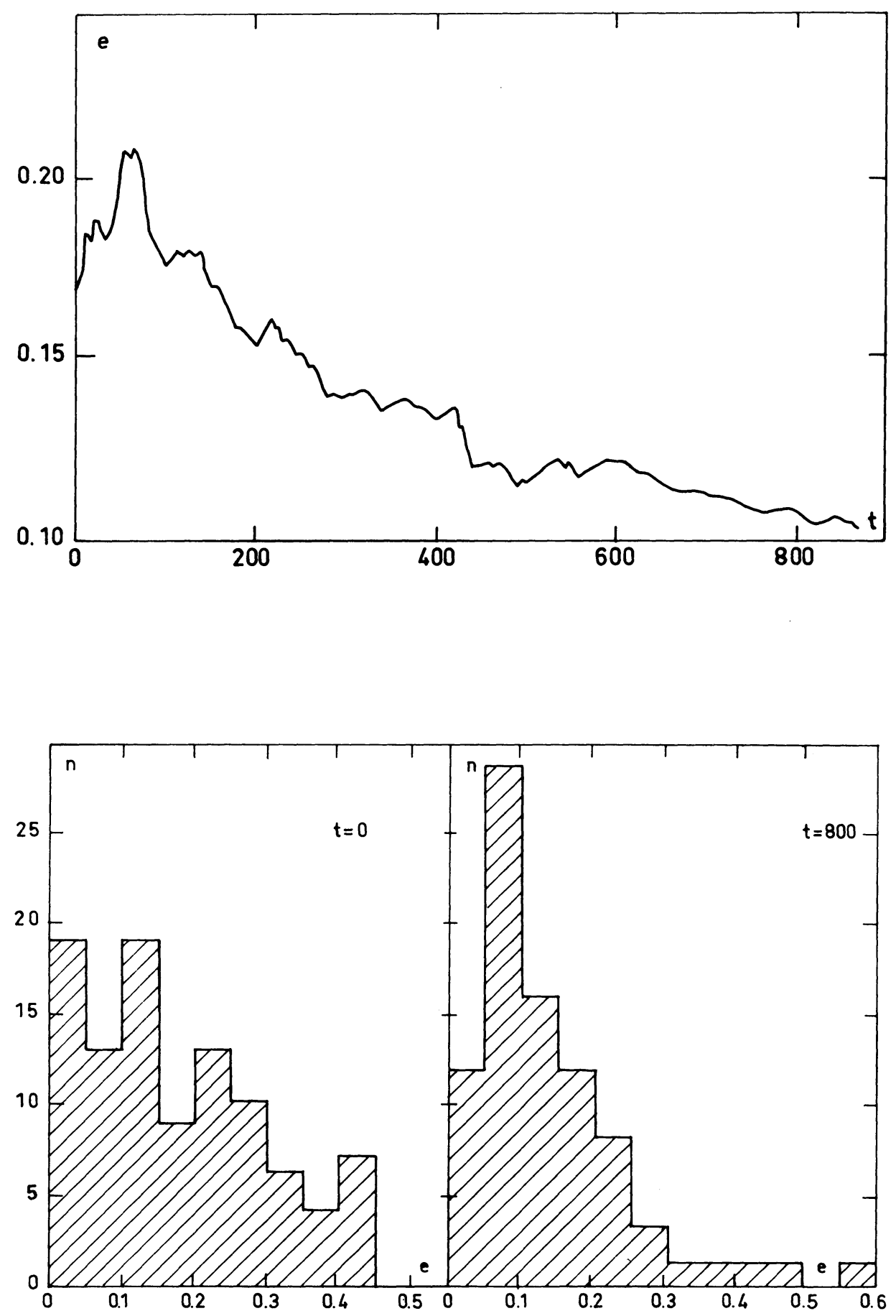

Fig. 4. The variation as a function of time of the mean excentricity of the orbits and the corresponding histograms. $k=0.1$. 

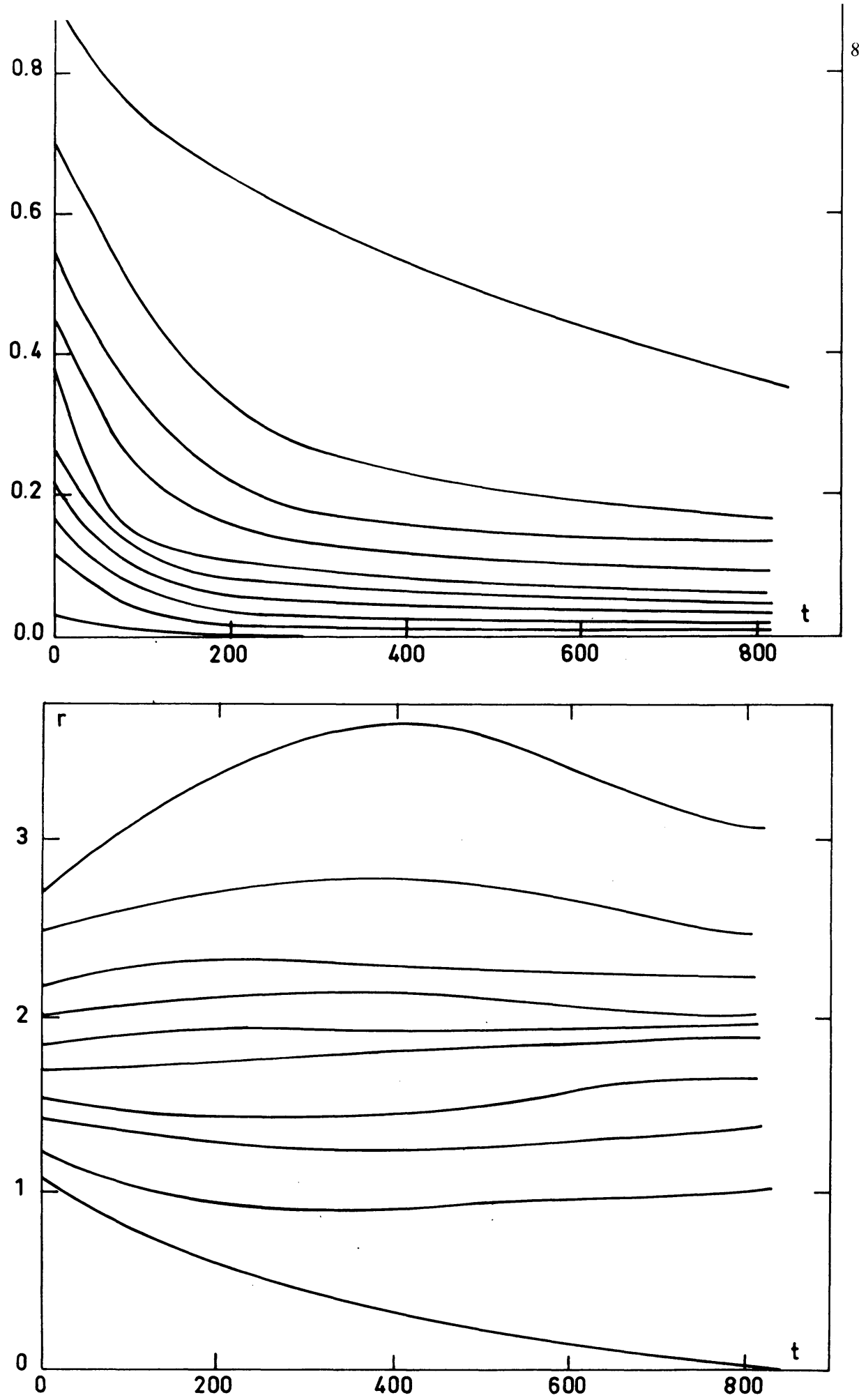

Fig. 5. Top: the ordinate represents the distance $|z|$ to the reference plane. At each time, the $N$ bodies are sorted in order of increasing $|z|$ and divided into ten groups. The curves represent the mean value of $|z|$ for each group. Bottom: same figure for the distance $r$ to the center. 

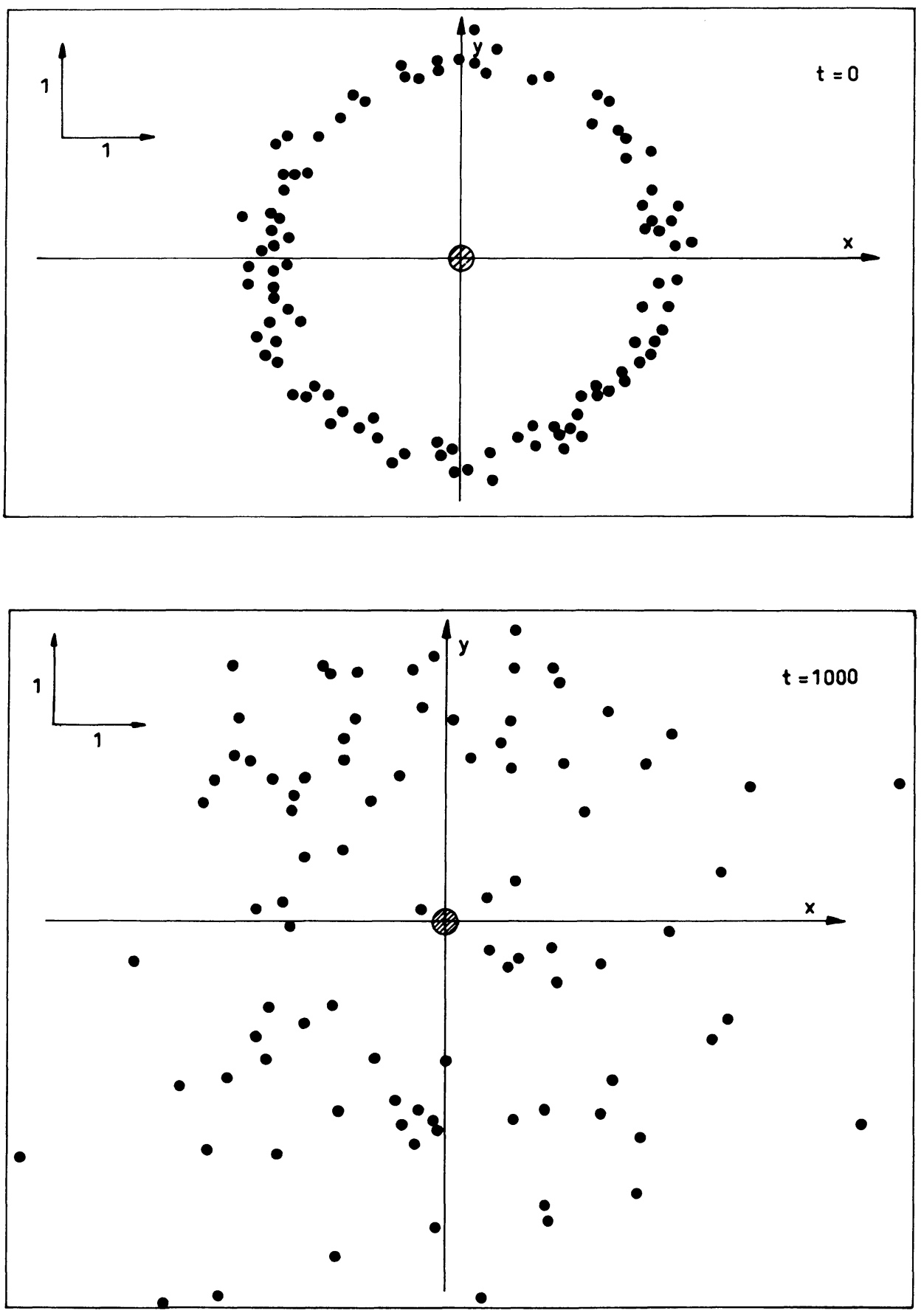

Fig. 6. Projections onto a plane perpendicular to the initial angular momentum vector. Initial trajectories are all ellipses lying between two spheres of radius 2 and 2.5 respectively centred on the central mass point. $k=0.3$. 

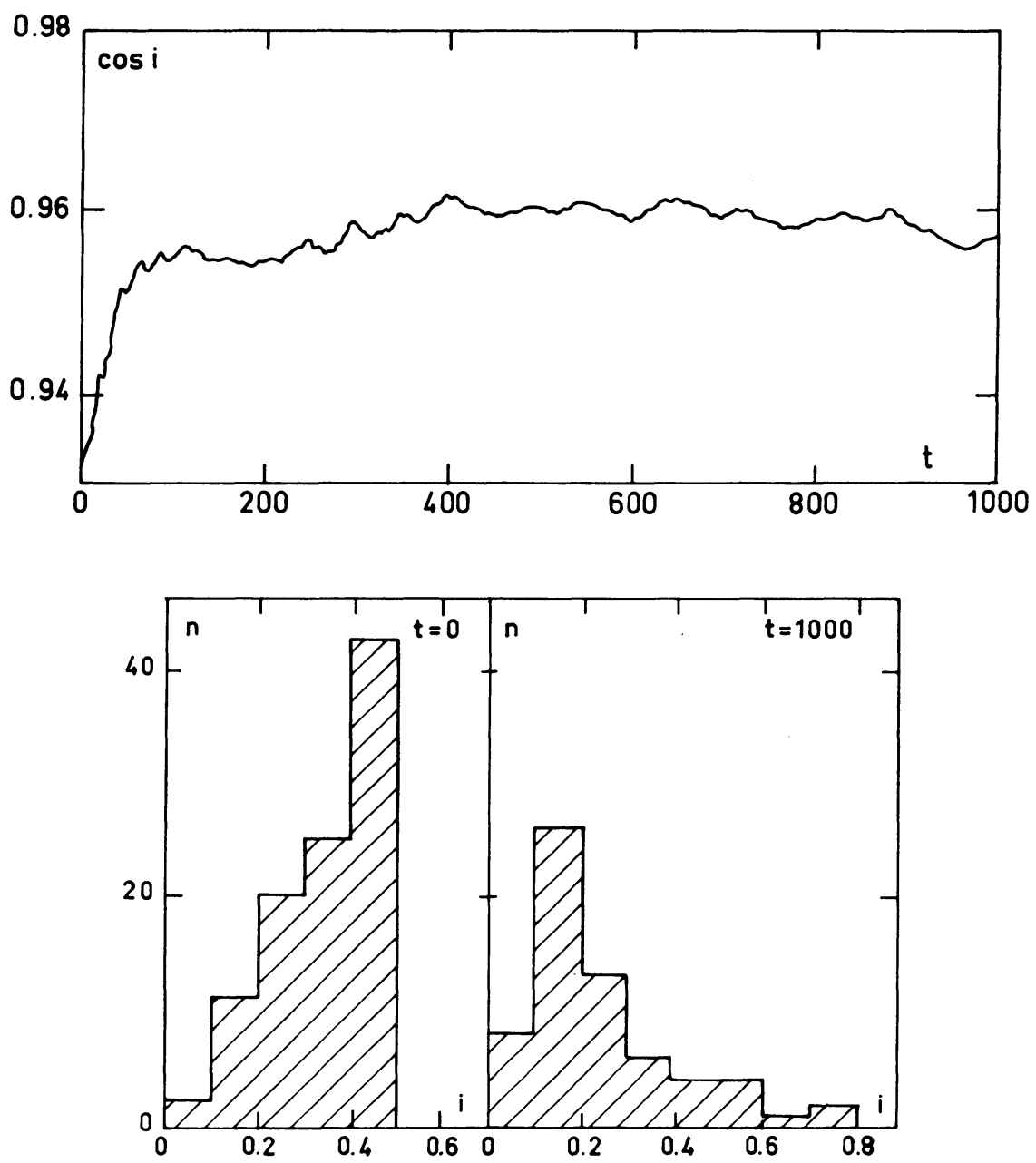

Fig. 7. Top: the variation as a function of time of the mean cosines of the inclination $i$. Bottom: the histogram of $i$ at the initial time and at the end of the computation. $k=0.7$.

physics because then the effect of each collision would be only local and the system would behave like a gas. On the other hand, a smaller frequency would increase computing time without changing fundamentally the physics.

Figures 1, 2, 3, 4 and 5 show an example of the evolution of the system. We can see on Figure 1 that the system has flattened considerably after 2500 collisions, but it is not yet completely flat. Figure 2 shows the same flattening in a more quantitative way. Figure 3 shows a spread on radius: the system extends. We can see on Figure 4 that, at first, the excentricities increase because a thermal equilibrium is established between radial and vertical velocities. After that the orbits tend to become more and more circular. Figure 5 shows the flattening and the concentration towards the centre in a more detailed way. Figures 6, 7, 8 show the evolution of the system for a different 
case. In the case of Figure 6, the smallest and the largest initial distances from the centre are 2 and 2.5 instead of 1 and 3 (Figure 3). The bodies are initially more concentrated in a thinner shell and the spread on radius is very important.

Alfvén and Arrhenius (1970a, b) have suggested that a planet is formed out of a jet stream. A jet stream is a group of bodies moving in similar elliptical orbits around a central body. Now, a given body will necessarily return to the point at which it last suffered a collision and since, moreover, partially inelastic collisions will tend
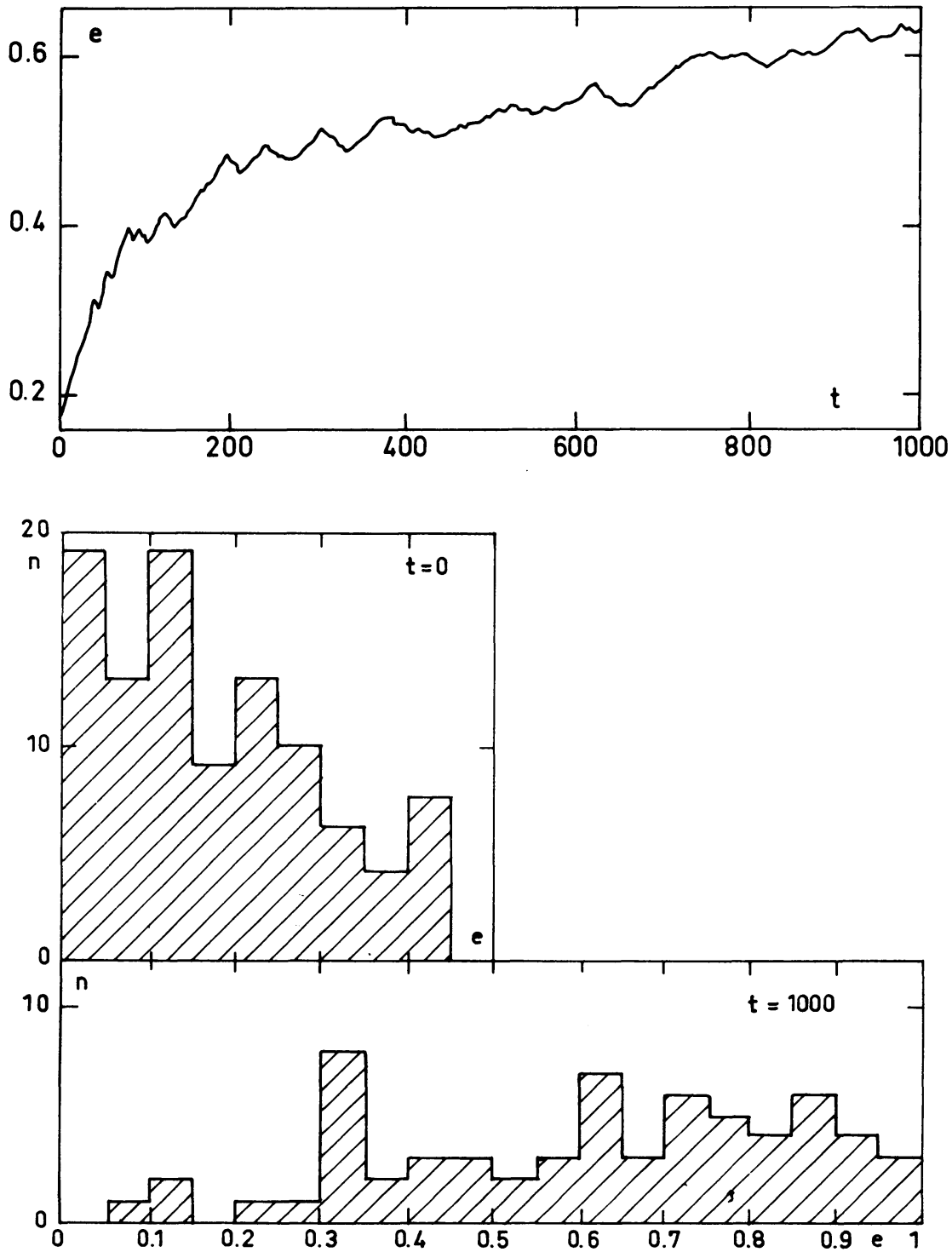

Fig. 8. The variation as a function of time of the mean eccentricity of the orbits and the corresponding histograms. $k=0.7$. 
to make orbits increasingly similar, it seems not unlikely that trajectories will become 'focused', and the circulating bodies will form themselves into a jet stream stable against external perturbations.

This theory of the formation of planets is very attractive; however, it is by no means clear that such a stream can actually be formed out of a more uniform distribution. After a collision between two bodies $A$ and $B$, if a third body intervenes and collides either $A$ or $B, A$ and $B$ do not collide again. Experiment does not indicate a focusing process.

In the case of Figures 7 and 8, the collisions are more elastic than in the case of Figures 2 and 4. The flattening process is not very efficient if the coefficient of elasticity is too large and the orbits are less circular.

Of course, this first model must be perfected. But the results already suggest (a) that the collision rate decreases with time and the system tends towards a final equilibrium state, and (b) that such a mechanism can flatten the system. The flattening is already important when each particle has suffered about ten collisions. It is a very fast evolution since probably each particle in the case of Saturn has suffered about one collision per day.

Subsequent models will include fragmentation and coalescence during each collision, and also the use of a more realistic central field taking into account the important flattening of Saturn. The effect of a satellite can also be easily included.

\section{References}

Alder, B. J. and Wainwright, T. E.: 1959, J. Chem. Phys. 31, 459.

Alder, B. J. and Wainwright, T. E. : 1960, J. Chem. Phys. 33, 1439.

Alfvén, H. and Arrhenius, G.: 1970a, Astrophys. Space Sci. 8, 338.

Alfvén, H. and Arrhenius, G.: 1970b, Astrophys. Space Sci. 9, 3.

Bobrov, M. S.: 1970, in A. Dollfus (ed.), Surfaces and Interiors of Planets and Satellites, Academic Press, London, New York, p. 377.

Brahic, A.: 1974, in J. R. Shakeshaft (ed.), 'The Formation and Dynamics of Galaxies', IAU Symp. 58, in press.

Brosche, P.: 1970, Astron. Astrophys. 6, 240.

Cook, A. F., Franklin, F. A., and Palluconi, F. D.: 1973, Icarus 18, 317.

Jeffreys, H.: 1947, Monthly Notices Roy. Astron. Soc. 107, 260.

MacCrea, W. H.: 1960, Proc. Roy. Soc. London A256, 245.

Poincaré, H. : 1911, Leçons sur les hypothèses cosmoniques, Hermann, Paris, Chap. 5, p. 86.

Rahman, A.: 1964, Phys. Rev. 136A, 405.

Sanders, R. H.: 1970, Astrophys. J. 159, 1115.

Spitzer, L. and Saslaw, W. C.: 1966, Astrophys. J. 143, 400.

Spitzer, L. and Stone, M. E.: 1967, Astrophys. J. 147, 519.

Trulsen, J.: 1972a, Astrophys. Space Sci. 17, 241.

Trulsen, J.: 1972b, Astrophys. Space Sci. 17, 330.

Trulsen, J.: 1972c, Astrophys. Space Sci. 18, 3.

Ulam, S. M.: 1968, Bull. Astron. 3, 265.

Urey, H. C.: 1972, Astrophys. Space Sci. 16, 311.

Verlet, L. : 1967, Phys. Rev. 159, 98.

Verlet, L. : 1968, Phys. Rev. 165, 201. 